\title{
Stabilizing Biped Walking on Rough Terrain based on the Compliance Control
}

\author{
Masaki Ogino, Hiroyuki Toyama and Minoru Asada
}

\begin{abstract}
In this paper, we propose a control system that changes the compliance based on the walking speed to stabilize biped walking on rough terrain. The proposed system does not use the inclination of the terrain. Instead, the system changes walking modes depends on its walking speed. In the downhill terrain, when the walking speed is increased, the stiffness of the ankle in the support phase is controlled so as to brake the increased speed. In the uphill terrain, when the walking speed is decreased, the stiffness of the waist joint is controlled and the desired trajectory for the supported leg is shifted so as not to falls down backward. To validate the efficiency of the proposed system, the stability of walking with the proposed system is examined in the two dimensional dynamics simulation. It is shown that the robot with the proposed system can walk in the more variable rough terrain and with the broader walking speed than without changing the stiffness of the joints.
\end{abstract}

\section{INTRODUCTION}

Biped walking algorithms are divided roughly into two categories; the model-based walking and dynamics based walking. In the former algorithm, the precise parameters of the robot and the environment are needed to calculate the control parameters such as zero moment point. However, this strategy needs a robot to sense the surface of the floor in advance precisely. Ohter groups realizes the walking on rough terrain with special mechanisms in the foots. Yamaguchi et al. [8] developed the foot mechanism with which a robot can senses the relative position and absolute inclined angle of the ground. With that mechanism, they realized the walking on the terrain with different levels in real time control. Hashimoto et al. [1] developed a parallel-linked biped walker with the semi-active adaptive ground mechanism that realizes the stable support area on the ground with small different levels. Both strategies extended in model-based approach realized the walking rough terrain only in very limited way; statical walking with special mechanisms in foots.

However, humans seem to realize rough terrain walking very different ways from these approaches. Human walking seems to utilize the dynamics of the body efficiently without precise sensing of the ground state [3]. The approach to realize a biped walking by using the dynamics of the body is called dynamics-based walking approach. Owaki et al. investigates the effect of the non-linear springs on robustness of passive running [5]. Taga et al. [6] proposed a CPG (Central Pattern Generator) for biped walking, and showed that it can realize the robust walking on the flat floor and

M. Ogino and M. Asada are with JST ERATO Asada Project, Yamada-oka 2-1, Suita, Osaka 565-0871, Japan \{ogino, a sada\} @ jeap.org

H. Toyama is with Department of Adaptive Machine Systems, Osaka University, Yamada-oka 2-1, Suita, Osaka 565-0871, Japan hiroyuki.toyama@ams.eng.osaka-u.ac.jp uniform slopes thanks to global entrainment of the body, control and environment dynamics. Miyakoshi [2] proposed the memory based control with which a robot can walk on known slope and the rolling slope. However, in these studies, the setting of the rough terrain is very limited and there have been few studies to investigate the possibility of dynamics based walking on rough terrain.

The rough terrain we treated in this paper has the random different levels and gradients that are relatively small to the robot body. On this terrain, the proposed controller enables a robot to walk stably without sensing the ground state by utilizing the compliance control.

In the following, first, the basic idea of the controller is introduced. Then, the effectiveness of the proposed controller is shown in the simulation experiments. Finally, the discussion and conclusion are given.

\section{WALKING ON ROUGH TERRAIN}

In the daily life, there are various types of surfaces in the ground. Depending on the difference of the levels or the tilting angle of the ground, human changes its walking pattern. Here, we classify the rough terrain into the two categories. The first one is the small rough terrain, in which the difference of the levels are relatively small compare to the size of the body. To stabilize the walking on this type of the terrain, the controller should has the feed back property to go back to the normal walking automatically. The second one is a large rough terrain like a staircase or a steep slope. For walking on this type of terrain, human should know the state of the terrain in advance by the visual information. Here, we treat the two dimensional walking for the first type of terrain, in which a robot can automatically recover its walking against the small disturbance.

One of the reasons for falling down during walking on rough terrain is the excess decrease or increase of the kinetic energy caused by the level difference. The proposed controller can compensate this energy disturbance so that it prevents a robot from falling down in forward or backward direction. In the following, first, the basic controller for walking in the flat floor is introduced. Then, the rough terrain whose difference of levels are small relative to the body are classified into the following three groups and the stabilizing control mode for each group on is introduced.

1) rough downslope: The angle of the inclination of the ground changes randomly, but always negative.

2) rough upslope: The angle of the inclination of the ground changes rondomly, but always positive. 
3) rough terrain: The angle of the inclination changes rondomly, positively and negatively.

\section{A. Base Control}

The basic walking controller changes the control method depending on the walking phase (Fig. 1). In the first of

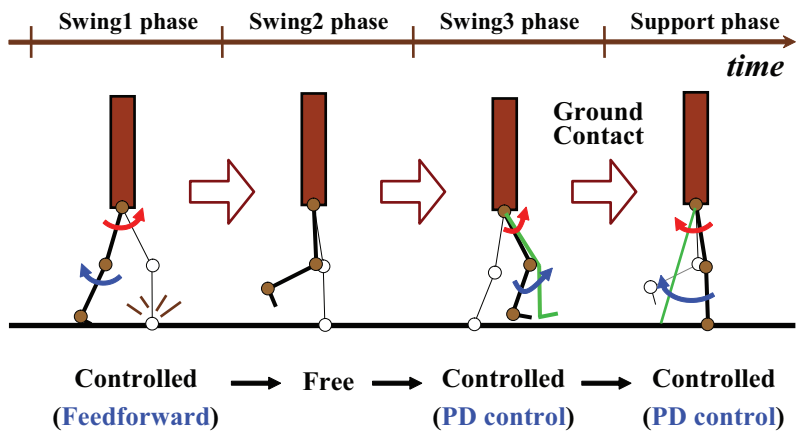

Fig. 1. Phase of walking

swing phase, the constant torque is applied to waist and knee joints of the swing leg. In the second phase, no torques are applied and the swing leg moves only by the inertial force. In the third phase, the proportional-derivative (PD) control is applied to the waist and knee joints to realize the landing posture that is determined in advance. In the stance phase, PD control is used to bring the stance leg backward. This control method can realize the torque pattern similar to the human walking [4].

The torque $\tau$ applied to the joint is given by the following equation,

$$
\tau=-K_{p}\left(\theta-\theta_{d}\right)-K_{v}\left(\dot{\theta}-\dot{\theta}_{d}\right)+\tau_{d},
$$

where $\theta, \theta_{d}, \dot{\theta}$, and $\dot{\theta}_{d}$ are the current and desired position and speed of the joint angle, and $K_{p}$ and $K_{v}$ are the gains for PD control, respectively. The desired angle in PD control $\theta_{d}$ is calculated by the following simple cosine function,

$$
\theta_{d}=\left\{\begin{array}{ll}
\frac{\theta_{f}-\theta_{0}}{2}\left(1-\cos \frac{\pi t}{T}\right)+\theta_{0} & (t<T) \\
\theta_{f} & (t \geq T)
\end{array},\right.
$$

where $\theta_{0}$ and $\theta_{f}$ are the initial and final angles of the joint in the phase, respectively, and $t$ and $T$ are the current time and the transition time to the next phase. This controller realize a stable walking in the flat terrain. However, on a rough terrain, a robot easily falls down. In order to realize the stable walking on the rough terrain, the compliance property is added to the control in the stance leg, as explained in the following section. The complicance (stiffness) of the joint angles are realized by changing the gains of PD control, $K_{p}$ and $K_{v}$.

\section{B. Control for Rough Downslope}

In the rough downslope, in which the inclination angle of the ground changes rondomly but the angles are always negative, the typical cause of falling down is the excess increase of the kinetic energy (Fig. 2). To suppress the

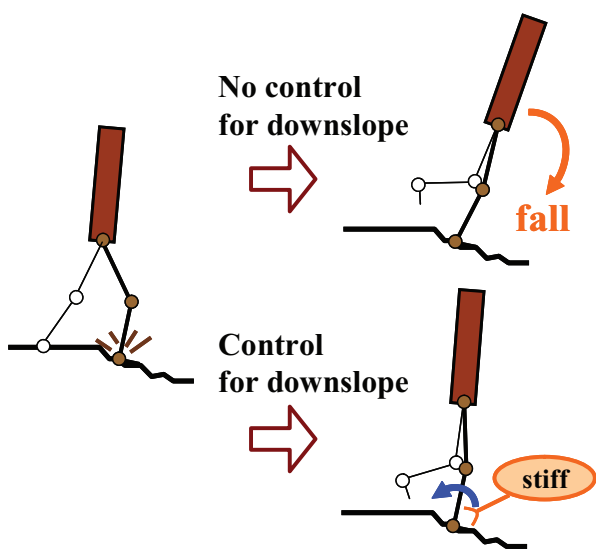

Fig. 2. Walking for downslope

increase of walking speed, the stiffness of the foot joint in the first stance phase is made high when the body of the speed exceeds certain threshold. The stiffness of the joint angle is determined by the gain of PD control, thus the control is simply given by the following equation,

$$
K_{p}=\left\{\begin{array}{ll}
K_{3 p}^{\text {down }} & \left(V>V^{\text {down }}\right) \\
K_{3 p} & \left(V \leq V^{\text {down }}\right)
\end{array},\right.
$$

where $K_{p}$ is the proportional gain of foot joint in the stance leg, $V$ is the walking speed of the body, $V^{\text {down }}$ is the threshold of the walking speed, and $K_{3 p}, K_{3 p}^{\text {down }}$ are the high and low constant values.

\section{Control for Rough Upslope}

The gain control in the rough upslope can be sumarized as Fig. 3.

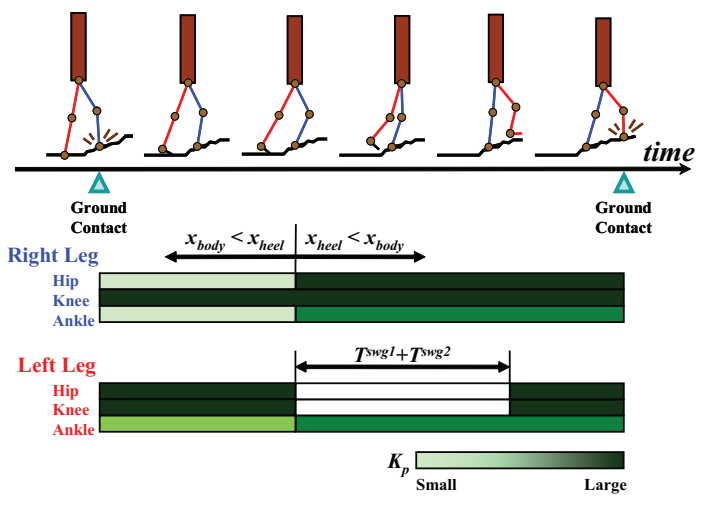

Fig. 3. Change of $K_{p}$ in upslope phase

In the rough upslope, in which the inclination angle of the ground changes rondomly but the angles are always positive, a robot falls down backward because of the excess decrease of the kinetic energy. To prevent the decrease of the kinetic energy, the desired joint angle of the conroller in the stance leg phase is changed when the walking speed is lower than the certain threshold. The stance leg phase is divided into the first half and the last half phases. 


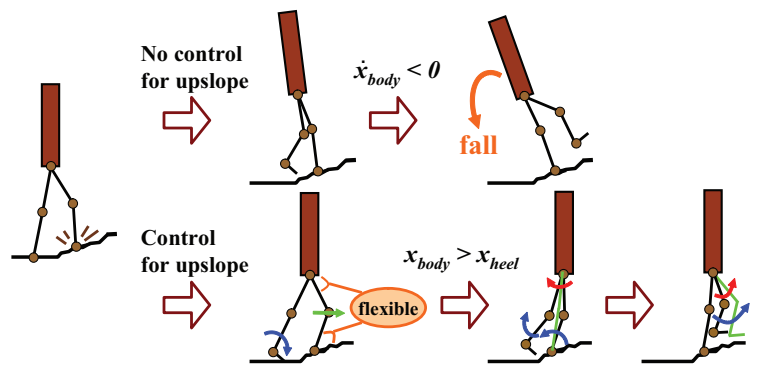

Fig. 4. Walking for upslope

1) control for the first half of the stance phase: When a robot is modeled by a simple inverted pendulum, walking is modeled as ascending the potential energy. When the initial speed is low and the kinetic energy is lower than the potential energy, the robot falls down backward. However, if the stance leg is made shorter and the hight of the center of mass is made lower, as mentioned Fig. 5 (b), the potential energy to get over becomes lower.
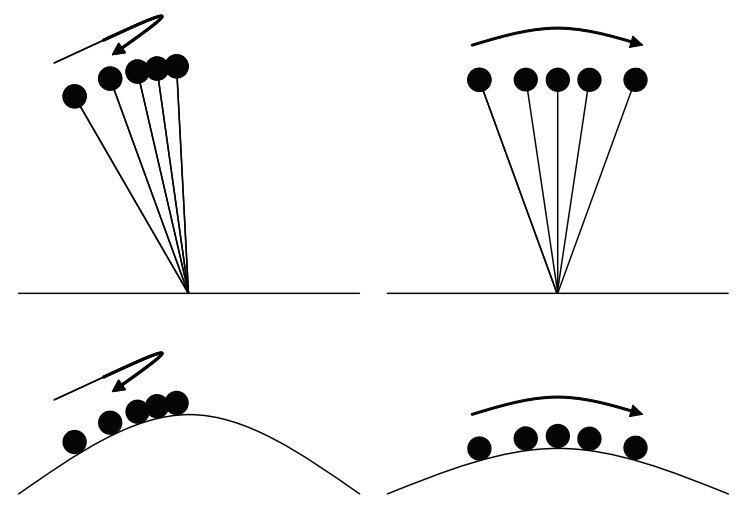

(a) Invariable expansion and (b) Variable expansion and concontraction of leg traction of leg

Fig. 5. Virtual inverted pendulum and potential

Thus, to make the height of the center of mass lower, when the walking speed becomes lower, the controller bends the knee joint more with high gain of PD control. However, the gain of the waist and foot joints are made lower. This lower stiffness enables a robot to keep the trunk upright and makes foot fit to the ground without the detail information about the rough terrain. When the vertical position of the body $x_{b o d y}$ proceeds the supporting point $x_{\text {heel }}$, the PD gains of waist and foot are got back to high gain.

2) control of the second half of the stance phase: When the walking speed is still low in the second half of the stance phase, further control is applied. Another solution to prevent the falling down backward is to increase the walking energy by extending the ankle joint in the second half of the stance phase. It is necessary to set the PD gain of the ankle joint not so high so that the weight shifts smoothly. Moreover, the desired posture at the end of the stance phase is also changed as the stance leg does not go so backward (to make the relative position of the body to the supporting point higher than usual.) Just after this control phase, the feed forward torques of the first swing phase that are applied to waist and knee joints are augmented than usual so that the swing leg contacts with the ground.

\section{Rough terrain}

The walking system for general rough terrain can be composed by integrating the controlers for rough upslope and downslope above mentioned. The integrated controller for one leg is given in Fig. 6. In this figure, Upslope and Upslope' are the walking modes for the first and second half of the stance leg in upslope, respectively. Whether the controller enters the control phase Upslope' depends on the walking speed, $V^{\prime}{ }_{h}$, when the opposite leg contacts with the ground. Thus, the controllers for right and left legs interacts each other as shown in Fig. 7.

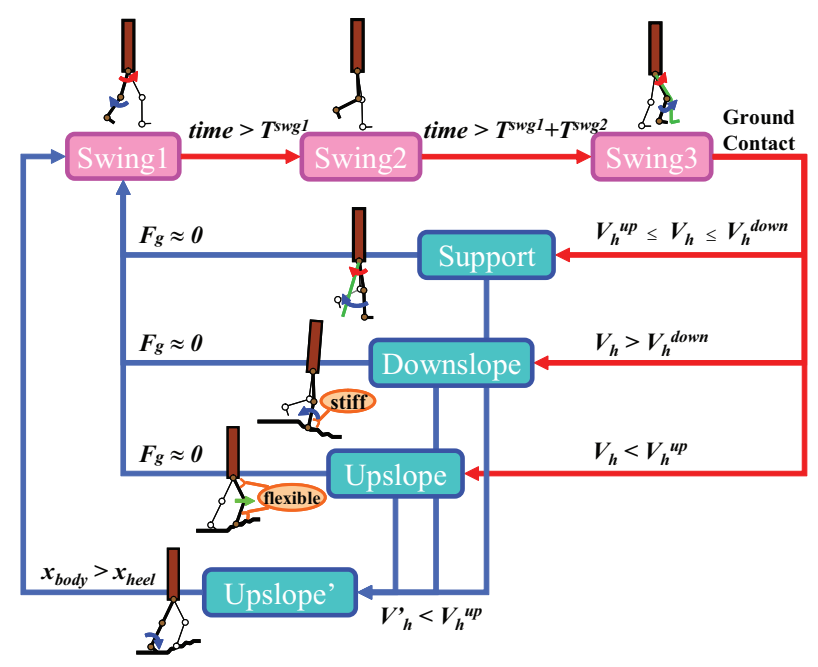

Fig. 6. Integration model for uneven surface

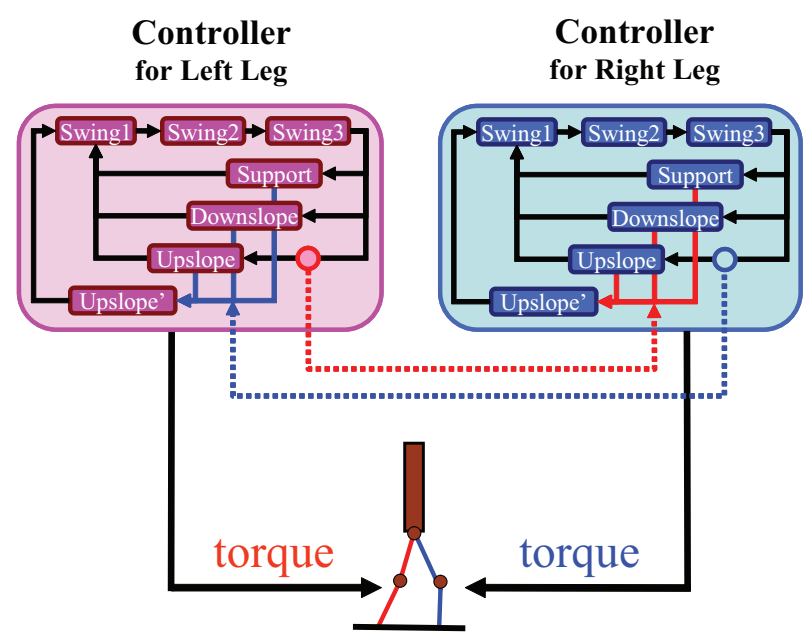

Fig. 7. Walking control system 


\section{EXPERIMENTAL RESULTS}

\section{A. Simulation setting}

1) Controller: Fig. 8 indicates the robot model used in the simulation. The robot consits of seven links: one upper body, two thighs, two shanks and two soles. The values of

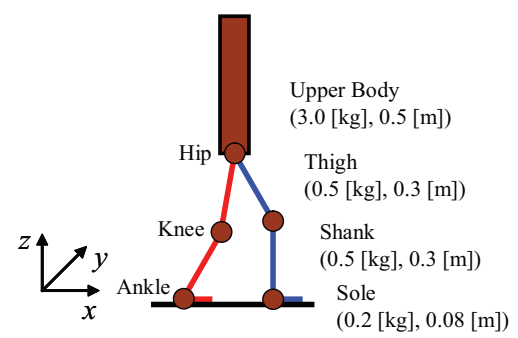

Fig. 8. Robot model

feedforward torques and PD gains in each control phase are shown in Table 1.

TABLE II

POSITIONS OF THE TARGET

\begin{tabular}{|c|c|c|c|c|c|c|c|}
\hline Joint & \multicolumn{2}{|c|}{ Hip } & \multicolumn{2}{|c|}{ Knee } & \multicolumn{2}{|c|}{ Ankle } & \\
\hline Parameter & $\theta_{f}$ & $\dot{\theta}_{f}$ & $\theta_{f}$ & $\dot{\theta}_{f}$ & $\theta_{d}$ & $\dot{\theta}_{d}$ & $T$ \\
\hline Swing1 & -23 & 0 & 20 & 0 & -10 & 0 & 0.2 \\
\hline Swing2 & -23 & 0 & 20 & 0 & -10 & 0 & 0.1 \\
\hline Swing3 & -23 & 0 & 20 & 0 & -10 & 0 & 0.15 \\
\hline Support & 20 & 0 & 0 & 0 & 0 & 0 & 0.5 \\
\hline Downslope1 & 20 & 0 & 0 & 0 & 0 & 0 & 0.5 \\
\hline Downslope2 & 20 & 0 & 0 & 0 & 0 & 0 & 0.5 \\
\hline Upslope1 & 20 & 0 & 40 & 0 & 0 & 0 & 0.3 \\
\hline Upslope2 & 20 & 0 & 0 & 0 & 0 & 0 & 0.3 \\
\hline Upslope' & 20 & 0 & 0 & 0 & $*$ & $*$ & 0.2 \\
\hline Upslope'1 & -30 & 0 & 40 & 0 & -25 & 0 & 0.2 \\
\hline Upslope'3 & -30 & 0 & 40 & 0 & -25 & 0 & 0.15 \\
\hline
\end{tabular}

$\tau_{d}[\mathrm{Nm}], K_{p}[\mathrm{Nm} / \mathrm{rad}], K_{v}[\mathrm{Nm} \mathrm{sec} / \mathrm{rad}], \theta_{f}[\mathrm{deg}], \theta_{d}[\mathrm{deg}], \dot{\theta}_{f}$ [deg/sec], $\dot{\theta}_{d}[\mathrm{deg} / \mathrm{sec}], T$ [sec].

Here, Downslope1 and Downslope2 in Fig. 6 are the control phases Downslope phase in case of $V>V^{\text {down }}$ and $V<V^{\text {down }}$, respectively. Upslope1 and Upslope2 are the control phases Upslope phase in case of $x_{\text {body }}<x_{\text {heel }}$ and $x_{\text {body }}>x_{\text {heel }}$, respectively. Upslope' 1 is the first swing phase Swing1 just after Upslope' phase. Upslop'3 is the third swing phase Swing3 just after Upslope' phase. The joint angles are set to 0 [deg] when the robot stands upright, and anticlockwise is set as the positive direction. In of Table $\mathrm{X} . \mathrm{X}$, the desired posutre in each phase $\theta_{f}=25[\mathrm{deg}]$ and $\dot{\theta_{f}}=0[\mathrm{deg} / \mathrm{sec}]$ are given.

The thresholds for phase transition in respective control phases are $V^{\text {down }}=0.6[\mathrm{~m} / \mathrm{sec}], V_{h}^{\text {down }}=0.85[\mathrm{~m} / \mathrm{sec}]$, and $V_{h}^{u p}=0.6[\mathrm{~m} / \mathrm{sec}]$. The PD gains to keep the trunk upright are $K_{w p}=5000[\mathrm{Nm} / \mathrm{rad}]$ and $K_{w v}=10[\mathrm{Nm}$ $\mathrm{sec} / \mathrm{rad}]$. The limitation of the torques in each joint is set as 10 [Nm].

The contact model between the robot and floor is modeled as spring-dumper interaction. Thus, the ground reaction forces, $F_{g x}$ and $F_{g z}$ are calculated as follows,

$$
\begin{aligned}
& F_{g x}=\left(-K_{g p}\left(x_{g}-x_{g 0}\right)-K_{g v} \dot{x_{g}}\right) g\left(-z_{g}\right), \\
& F_{g z}=f\left(-K_{g p} z_{g}-K_{g v} \dot{z_{g}}\right) g\left(-z_{g}\right), \\
& f(x)=\max (0, x), \\
& g(x)= \begin{cases}1 & (0.01<x) \\
100 x & (0 \leq x \leq 0.01) \\
0 & (x<0)\end{cases}
\end{aligned}
$$

where $K_{g p}(=2000[\mathrm{~N} / \mathrm{m}])$ and $K_{g v}(=100[\mathrm{~N} \mathrm{sec} / \mathrm{m}])$ are the proportional and derivative gains, respectively. $x_{g 0}$ is the first contact position in the horizontal direction. $x_{g}$ and $z_{g}$ are the positions in the horizontal and vertical positions, respectively. $\dot{x}_{g}$ and $\dot{z}_{g}$ are the velocities in the horizontal and vertical positions, respectielly.

2) Terrain with one even step: To validate the effect of the controller for upslope and downslope, the recovery after the walking on the one even step are examined. Fig. 9 shows the time course of walking speed during one steps. The horizontal axis indicates the walking time from the swing leg contacts with the ground. Everytime the swing leg contacts with the ground, the time is set to 0 . The vertical axis indicates the speed of the body. For the down step (Fig. 9(a)), the walking speed just after the down step becomes higher (B) than that on the flat floor (A). However, it is shown that the walking speed converges to the normal speed within a few steps. For the up step (Fig. 9(b)), the walking speed just after the up step becomes lower (B) than that on the flat floor. The walking speed goes higher by the controller (C). However, it converges to the normal speed within a few steps.

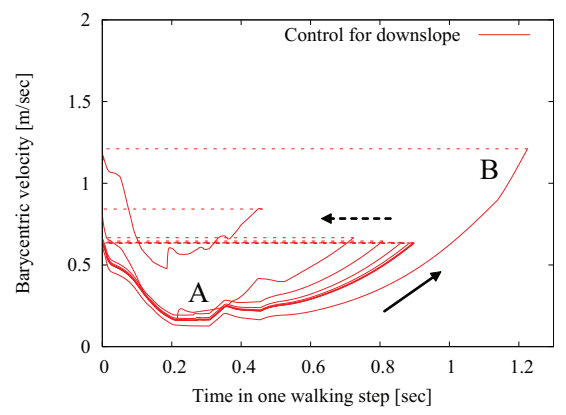

(a) Walking velocity for down step

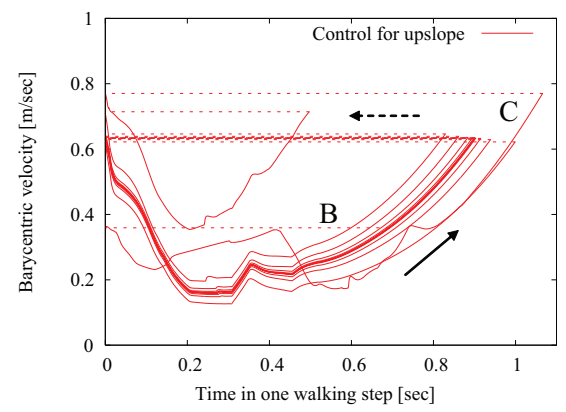

(b) Walking velocity for up step

Fig. 9. Walking speed on the terrain with one even step 
TABLE I

TORQUES AND GAINS

\begin{tabular}{|c|c|c|c|c|c|c|c|c|c|}
\hline Joint & \multicolumn{3}{|c|}{ Hip } & \multicolumn{3}{|c|}{ Knee } & \multicolumn{3}{|c|}{ Ankle } \\
\hline Parameter & $\tau_{d}$ & $K_{p}$ & $K_{v}$ & $\tau_{d}$ & $K_{p}$ & $K_{v}$ & $\tau_{d}$ & $K_{p}$ & $K_{v}$ \\
\hline Swing1 & 1.0 & 0 & 0 & 1.0 & 0 & 0 & 0 & 50 & 0.1 \\
\hline Swing2 & 0 & 0 & 0 & 0 & 0 & 0 & 0 & 50 & 0.1 \\
\hline Swing3 & 0 & 300 & 0.1 & 0 & 300 & 0.1 & 0 & 50 & 0.1 \\
\hline Support & 0 & 300 & 0.1 & 0 & 300 & 0.1 & 0 & 50 & 0.1 \\
\hline Downslope1 & 0 & 300 & 0.1 & 0 & 300 & 0.1 & 0 & 230 & 0.1 \\
\hline Downslope2 & 0 & 300 & 0.1 & 0 & 300 & 0.1 & 0 & 50 & 0.1 \\
\hline Upslope1 & 0 & 3 & 0.01 & 0 & 300 & 0.1 & 0 & 3 & 0.01 \\
\hline Upslope2 & 0 & 300 & 0.1 & 0 & 300 & 0.1 & 0 & 50 & 0.1 \\
\hline Upslope' & 0 & 300 & 0.1 & 0 & 300 & 0.1 & 0 & 5.5 & 0.1 \\
\hline Upslope'1 & 1.5 & 0 & 0 & 1.5 & 0 & 0 & 0 & 50 & 0.1 \\
\hline Upslope'3 & 0 & 300 & 0.1 & 0 & 300 & 0.1 & 0 & 50 & 0.1 \\
\hline
\end{tabular}

3) Walking on down and up slope: The rough down and up slope are constructed by the polygonal lines. The The $i$ th ground position $(X(i), Z(i))$ is defined by the following equations,

$$
\begin{aligned}
& X(i)=X(i-1)+X_{0} R \\
& Z(i)=Z(i-1) \pm Z_{0} R
\end{aligned}
$$

where $R$ is the random number from 0 to $1 . X_{0}$ and $Z_{0}$ are the constant values that determines the degree of the roughness of the ground.

Figs. 10 and 12(b) show 11(b)

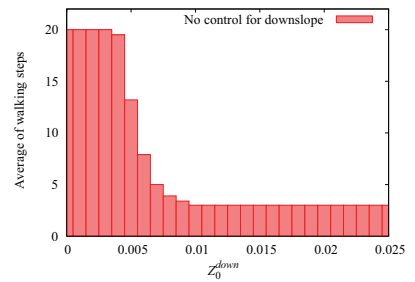

(a) No control for downslope

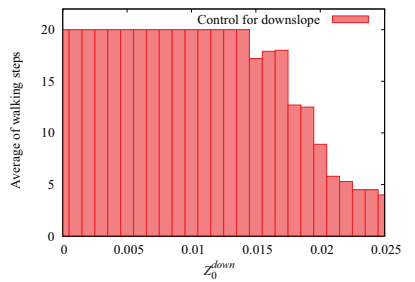

(b) Control for downslope
Fig. 10. Average of walikng steps for downslope

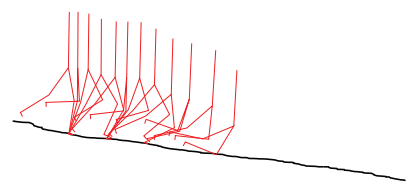

(a) No control for downslope

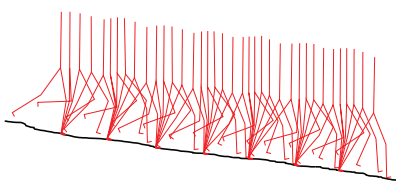

(b) Control for downslope
Fig. 11. Transition of walk for downslope

4) Walking on rough terrain: The rough terrains used in the simulation are constructed by the polygonal lines. The $i$-th ground position $(X(i), Z(i))$ is defined by the following equations,

$$
\begin{aligned}
& X(i)=X(i-1)+X_{0} R \\
& Z(i)=Z(i-1)+Z_{0}(R-0.5)
\end{aligned}
$$

where $R$ is the random number from 0 to $1 . X_{0}$ and $Z_{0}$ are the constant values that determines the degree of the roughness of the ground.

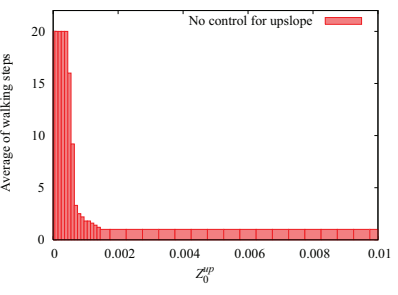

(a) No control for upslope

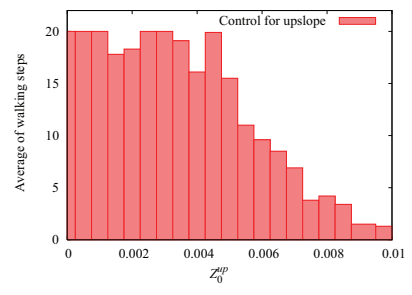

(b) Control for upslope
Fig. 12. Average of walking steps for upslope

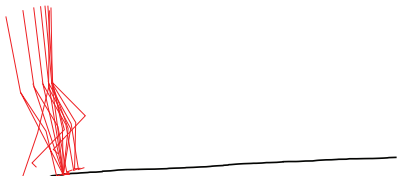

(a) No control for upslope

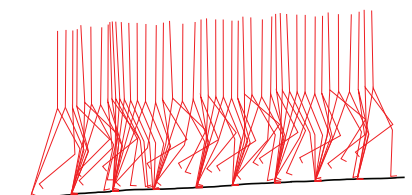

(b) Control for upslope
Fig. 13. Transition of walk for upslope

Fig. 14(a) shows the averaged walking steps with and without the proposed controller in relation to the roughness degree, $Z_{0}$ and $X_{0}=0.05[\mathrm{~m}]$. The number of maximam steps is 20 and 10 trials are examined in each roughness. The graph shows the robustness improves compared with the walking without the proposed controller. Fig. 14(b) is the time sequences of the walking with and without the proposed controller when the roughness of the ground is set as $X_{0}=0.05$ and $Z_{0}=0.023[\mathrm{~m}]$.

\section{CONCLUSION}

This paper proposes a walking controller that enables a robot to walk on the rough terrain by changing the compliance of joints without sensing the state of the surface of the ground.

To apply the proposed controller to a real robot, there are several problems to be solved. First, the current control model is restricted to two dimensional. The motions on the frontal plane affects to that on the sagittal plane. It is the next challenge to develop the controller for the stabilization of the motions on the sagittal plane. The second problem is the slips on walking. The current simulator does not consider the slips 


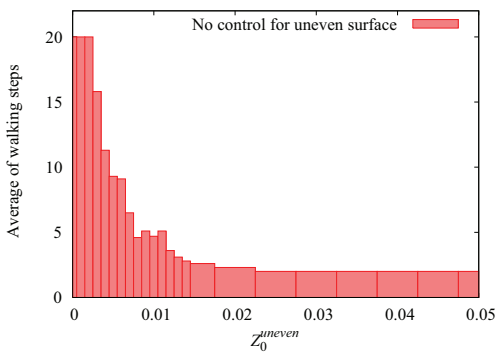

(a) No control for uneven surface

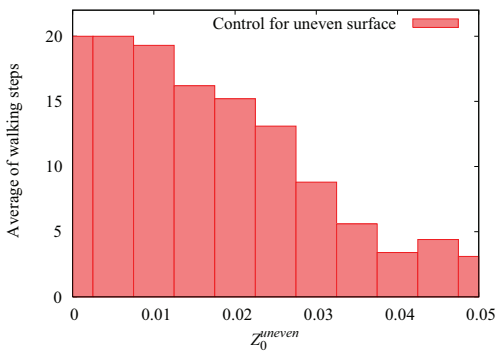

(b) Control for uneven surface

Fig. 14. Average of walking steps for uneven surface

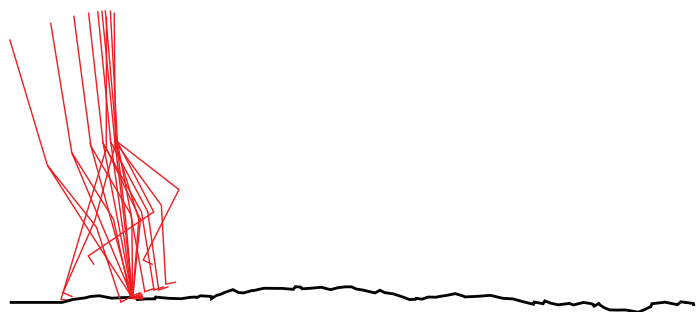

(a) No control for uneven surface

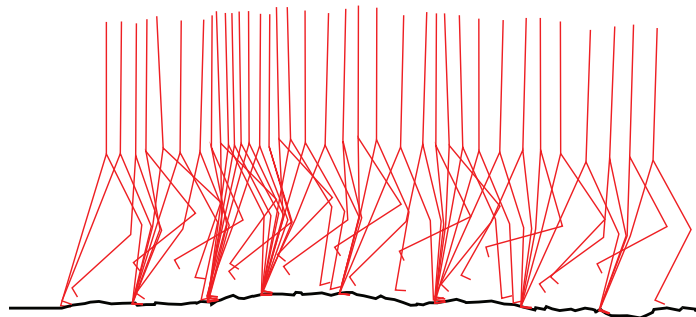

(b) Control for uneven surface

Fig. 15. Transition of walk for uneven surface

between the feets and the ground. In the real situation, the steeper the slope is, the more the robot slips. Third, the most difficult problem is the actuators. In this paper, we modeled the simple PD controller and the various types of the stiffness are realized by changing the gains of PD controller. However, the real DC motors that are usually used in humanoid robots are difficult to realize the low stiffness. Moreover, it is difficult to design the robot that utilizes the dynamics of the body with DC motors because power/weight ratio of DC motors are not suitable for low stiffness. The pneumatic actuators may be the possible candidates for dynamics based humanoid robots [7].

\section{REFERENCES}

[1] K. Hashimoto, H. O. Lim, and A. Takanishi, "Develoopment of foot system of biped walking robot capable of maintaining four-point contact," in Proceedings of the 2005 IEEE/RSJ International Conference on Intelligent Robots and Systems, 2005, pp. 1464-1469.

[2] S. Miyakoshi, "Memory-based bipedal walking control on the slope and uneven surface," in Proceedings of the Annual Conference of the Robotics Society of Japan (in Japanese), 2006, p. CDROM.

[3] S. Mochon and T. A. McMahon, "Ballistic walking," Journal of Biomechanics, vol. 1, no. 46, pp. 9-14, 2002.

[4] M. Ogino, K. Hosoda, and M. Asada, "Learning energey-efficient walking with ballistic walking," in Adaptive Motion of Animals and Machines, H. Kimura, K. Tsuchiya, A. Ishiguro, and H. Witte, Eds. Springer-Verlag Tokyo, 2006, pp. 155-164.

[5] D. Owaki and A. Ishiguro, "Enhancing stability of a passive dynamic runnning biped by exploiting a nonlinear spring," in Proceedings of the IEEE/RSJ International Conference on Intelligent Robots and Systems, 2006, pp. 4923-4928.

[6] G. Taga, "A model of the neuro-musculo-skeletal system for human locomotion i emergence of basic gait," Biological Cybernetics, vol. 73, 1995.

[7] T. Takuma, K. Hosoda, M. Ogino, and M. Asada, "Stabilization of quasi-passive pneumatic muscle walker," in Proceedings of the Fourth International Symposium on Human and Artificial Intelligence Systems, 2004, pp. 370-375.

[8] J. Yamaguchi, N. Kinoshita, A. Takanishi, and I. Kato, "Development of a dynamic biped walking system for humanoid -development of a biped walking robot adapting to the humans' living floor-," in Proceedings of the 1996 IEEE International Conference on Robotics and Automation, 1996, pp. 232-239. 